\title{
LOS UNIVERSALES Y EL PROBLEMA DEL CONCEPTO EN EL MEDIOEVO
}

En 1939, en un trabajo presentado a la Preussische Akademie der Wissenschaften, N. Hartmann se planteó al final la siguiente cuestión: wo kommt denn schliesslich das Wissen um den Begriff auf? (¿dónde aparece pues por

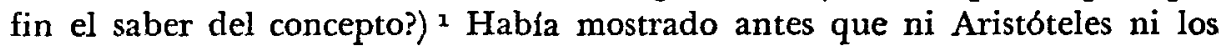
antiguos supieron acerca del concepto, esto es, que la actitud de ellos con respecto a las ideas generales y a los universales era enteramente realista: el universal, como el eidos y el génos, pertenece a la realidad.

El trabajo que presento intenta dar respuesta a la interrogante de Hartmann. No es pretensión mostrar algo enteramente nuevo. Que a fines de la Edad Media, en la Baja Escolástica, se tuvo ya un saber del concepto, es algo que el mismo Hartmann no ingoraba, y antes, en 1910, K. Michalski habia publicado fragmentos de manuscritos de Ricardo de Mediavilla (+ 1308) y de Herveo Naral (+ 1323) por los que nos enteramos que estos escolásticos tenían una idea cabal del concepto, entendido éste como una estructura mental de naturaleza enteramente diferente del acto de entender. Al concepto le llamaron esse objectivum por oposición al esse subjectivum. Ahora bien, esse subjectivum es lo que existe realmente sea como substancia o como accidente. El acto de entender es un esse subjectivum al que se opone en el mismo acto el esse objectivum. ${ }^{2}$

Nuestra tarea se circunscribe entonces a dos puntos: $x$ ) ver si hubo un saber acerca del concepto antes del siglo xIv, 2) por qué es que esa idea o saber acerca del concepto fue aceptada recién en el siglo xIv coincidentemente con las postrimerías del pensamiento escolástico.

Michalski ${ }^{3}$ ha señalado al conceptualismo como uno de los factores disolventes de la Escolástica. En verdad, el conceptualismo es sólo un sintoma de una crisis más profunda: la crisis del realismo medieval. Averiguar entonces por las condiciones en que es posible o aceptable el conceptualismo es de paso preguntarse por las razones del colapso del realismo medieval.

Pero colapso no es muerte. $\mathrm{Y}$ el cristianismo no podría ser sin una suerte de realismo. Si el escepticismo del siglo xrv —al que inevitablemente va a parar el conceptualismo- disuelve definitivamente las categorfas básicas del pensamiento medieval, a saber: la idea de "ordo" y la concordancia entre

1 Aristóteles u. das Problem des Begriffs. En Kleinere Schriften Bd. II Berlin 1957, pp. 100-129. Hay tr. castellana. México.

2 Michalski, Les courants philosophiques d Oxford et d Paris pendant le xiv siècle, Cracovie, 1910.

3 Op. cit. 
la fe y la razón, ese escepticismo es sin embargo fideista: se asienta en última instancia en la convicción de la potentia Dei absoluta. Dentro de la perspectiva de una teologia explicada por la potentia Dei absoluta Ockham estructura una metafísica de corte individualista en la que toda diferencia formal o de razón se reduce a una diferencia real de cosas realmente separadas o realmente separables: numquam potest esse distinctio conceptuum nisi propter aliquam distinctionem a parte rei. (In IV Sent. Prol. q. II, LL). En esta metafísica individualista ya no es posible ni siquiera el conceptualismo sensu stricto, esto es el conceptualismo lógico. El nominalismo de Ockham, que dista ya del conceptualismo lógico, aparece entonces como un medio de superación del realismo tradicional, del realismo de los universales, pero para fundar un nuevo realismo: el realismo individualista.

Veamos cómo evoluciona el realismo de los universales desde la Temprana Escolástica. La primera dificultad que nos sale al paso es el hecho de que en el Medioevo antes del siglo xiv no sólo ha habido realismos de los más diversos matices sino también nominalismos y hasta conceptualismos, esto último cuando menos en un caso. El tratamiento de esta dificultad lo dejamos para después, luego de haber descrito el cuadro del realismo de los universales.

El realismo de los universales supone que la estructura de la realidad está constituida de relaciones esenciales y que hay una dependencia efectiva del individuo a la esencia y hasta de la especie al género. Además, que la realidad ha sido creada de acuerdo a arquetipos en la mente divina y que, en consecuencia, es plenamente inteligible, aunque no quoad nos. Esta metafísica neoplatónico-cristiana va a tener vigencia a lo largo de toda la Escolástica. La introducción del Aristóteles metafísico en el siglo xirr sirvió sólo para reforzarla. Dentro de este cuadro la tendencia más natural fue el realismo: la suposición de que los géneros, las especies y los individuos son realidades o pertenecen a la realidad.

El realismo tiene un proceso de evolución que va desde posiciones extremas hasta posiciones moderadas. Hay pensadores de la Temprana Escolástica, como J. Escoto Erigena, Anselmo de Aosta, G. de Champeaux y algunos representantes de la Escuela de Chartres, para quienes los géneros y las especies son realidades en sí con mayor o igual peso ontológico que los individuos. Este realismo exagerado - que tiene sin embargo poco que ver con el realismo de las ideas de Platón * - parece extinguirse desde el segundo cuarto del

4. Salvo el caso singular de Bernardo de Chartres, de cuya doctrina sólo sabemos por J. de Salisbury (Metal. II, 17), quien lo llamó por eso "el más perfecto platónico de su tiempo". Bernardo afirma que las especies son ideas divinas, ejemplares eternos, de las que derivan las formae nativae que al combinarse con la materia forman el mundo transitorio de las cosas sensibles. En todas las otras modalidades de realismo extremo se afirma la inherencia o inmanencia en las cosas singulares de las formas genéricas y específicas, sosteniéndose que éstas constituyen la realidad esencial aunque universal de las cosas y que las 
siglo xir. En efecto, Juan de Salisbury (1115-1180) cuyos escritos constituyen el testimonio intelectual más importante del siglo xIr, menciona hasta diez soluciones al problema de los universales, y de éstas sólo una perteneciente a lo que podría llamarse la tendencia realista extrema, que la atribuye a Bernardo de Chartres, pero que en la segunda mitad del xII ya nadie la defiende: sed pridem hanc nullus profitetur (Metologicus, II, 17). En el Polycraticus, VII, 12, escrito en 1159, J. de Salisbury enumera sólo cuatro soluciones, de las que una es abstraccionista, otra intelectualista y dos nominalistas, y agrega que buscar los géneros y las especies como si fuesen cosas es tan vano como buscar la substancia de la solidez en la sombra de los cuerpos.

Desde la segunda mitad del siglo xII, y sobre todo en el siglo xiIr, la opinión dominante es la de que el universal no es cosa, pues sólo existen los individuos. ${ }^{5}$ Sin embargo, al universal sigue considerándosele componente esencial y causa formal del individuo. En el individuo se funda la igualdad esencial de las cosas, pero esa igualdad se define en el entendimiento. De modo que el universal no está propiamente en las cosas sino en el entendimiento, pero son las cosas el fundamento real de esa universalidad. En otras palabras: el universal está virtualmente en las cosas, pero en acto, esto es como predicado de muchos, sólo en el entendimiento. Es a esta solución a lo que se ha dado en llamar realismo moderado. Hay fundamentalmente dos variantes en esta posición: r) La que afirma que el conocimiento del universal se debe a una comunidad de estructura entre la mente y lo real y que se expresa en la fórmula: el ser es la medida del entendimiento y el entendimiento es la medida del ser. 2) La que postula que el entendimiento es una simple facultad enteramente diferente de la cosa antes de conocerla, pero que deviene semejante a ella en el acto de conocerla. En ambos realismos se establece una teoria de la abstracción explicativa del proceso del conocimiento del universal. Se parte del supuesto de que las cosas son de un modo en el ser y de otro en el conocer: el modus essendi es diferente del modus cognoscendi. En el ser son de modo singular y en el entendimiento de modo universal. Ello no significa que el entendimiento aporte algo nuevo o diferente sino simplemente que actualiza lo que está virtualmente en las cosas sea como principio o como común cualidad.

En el primer tipo de realismo se afirma que lo abstracto determina lo

cosas singulares sólo se diferencian accidentalmente. La realidad esencial de los individuos viene a ser su universalidad. Cfr. C. H. Haskins, The Renaissance of the 12th. Century, N. Y,. 1957, P. 344.

5 The "universals" controversy was practically decided before the thirteenth century: scholasticism unanimously accepted the solution arrived at the twelth. "The individual is the real substance: the universal derives its ultimate form from the subjetive work of our minds". M. De Wulf, Scholastic Philosophy, Nueva Xork, 1956, p. 105. 
concreto y que las cualidades fundamentan el ser individual.b. De esta manera, la mente, que opera con abstracciones, conoce directamente lo universal, que opera como principio de las cosas, por esa comunidad de estructura que se supone entre la mente y lo real. De alli que se afirme con Gilberto de Poitiers que los principios de la ciencia son los principios de las cosas mismas y que el universal, que es sólo una abstracción de las formas nativas en los individuos, se funde en la semejanza esencial de las cosas, las que existen realmente en sus modalidades individuales.

Con la introducción de la física y la metafísica aristotélicas en el mundo medieval cristiano, cosa que ocurre recién a fines del siglo xIr, el realismo se reformula en una teoría de la abstracción más precisa. Las categorías de acto y potencia son entonces claves para aclarar el proceso del conocimiento de lo universal. Al entendimiento se le concibe no como un elemento activo sino más bien pasivo: es una facultad (vis) en la que se realizan las virtualidades inteligibles de las cosas, que en sí y en acto son sensibles y singulares. No es que lo abstracto determine lo concreto, como en Gilberto de Poitiers. El entendimiento es más, bien una tabula rasa en la que nada hay escrtio. La cosa concreta a la luz del entendimiento agente deviene inteligible en acto y plasma su naturaleza inteligible en el entendimiento pasivo $y / 0$ posible, el que deviene también en acto por esa forma inteligible. El entendimiento en acto es la cosa inteligible en acto. El universal, que es un predicado que conviene a muchos (un nombre común) es también y en primer lugar la forma intelligibilis o species intelligibilis que aunque concebida sin materia en el entendimiento es con todo la que existe en la cosa como acto de ella, si bien en su modalidad individual. Como a forma o especie que es igual en muchos individuos le conviene empero un nombre propio: el nombre de la esencia.

Lo que importa destacar para nuestros propósitos es que en ambos tipos de abstracción se postula que el entendimiento es sólo un plano de realizaciones de lo real. Las formas abstraídas son a la par formas de conocimiento y principios del ser. Se aprecia pues una confusión entre lo lógico y lo ontológico, con preferencia neta por la explicación ontológica. Ahora, si entendemos el concepto como aquello de lo que la mente es consciente pero que es diferente aunque no independiente de ella, podríamos concluir que el pen-

6 Gilberto de Poitiers busca la explicación de lo concreto por lo formal, incluso hasta límites que el Concilio de Reims (1148) juzgó peligrosos de herejła. Hayen interpretando el pensamiento del obispo de Poitiers escribe: "....Les choses sont comme je les pense; or je les pense concrétes, mais á l'aid de concepts abstraits. Les choses sont donc en realité déterminées par des formes abstraites qui les constituent" (Le Concile de Reims et l'erreur theologique de Gilbert de la Porrée. Archives d'Histoire doctrinale et Litteraire du M. Age, t. X, p. 69).

Deus non est divinitas, sed divinitate, album est albedine... expresa la tendencia de Gilberto de explicar lo cuantitativo por lo cualitativo, lo individual por lo formal, lo existencial por lo esencial. Dios mismo estaría ontológicamente supeditado a su divinidad: 
sador medieval antes del siglo xIv no sólo no pudo ser conceptualista sino que ignoró acerca del concepto. $\mathrm{La}$ abstracción conduce indudablemente a la formación de conceptos, pero el pensador medieval no toma lo abstraído como una estructura de contenido lógico sino como una forma de lo real. La species intelligibilis, que es el acto mismo de entender, según hemos visto, no es lo que se conoce (no es objeto de conocimiento) sino aquello por lo que se conoce. Ahora bien, lo que se conoce es la cosa misma y no una estructura lógica, un esse objectivum como dirían los escolásticos del siglo xiv. Entonces, no hay aquí idea del concepto. En el realismo que supone comunidad de estructura entre lo mental y lo real se aprecia aún menos el concepto: el entendimiento es allí una apertura a todo el proceso de lo real. Podría argüirse quizá que el hecho de distinguir el modo de ser de una cosa diferente del modo de conocerla implica ya el conocimiento de la distinción entre lo lógico y lo ontológico, entre lo mental como signo de lo real y lo real como la cosa signada. Pero no es así. La distinción entre el modus essendi y el modus cognoscendi postula sólo que el entendimiento separa lo que en la realidad está unido y une lo que en la realidad está separado. En todo caso esto sólo significa que la realidad es de un modo en el ser y de otro modo en el conocer. Y en èl conocer está según el modo de ser del cognoscente: $\operatorname{cog}$ nitio fit, secundum quod cognitum aliquo modo est in cognoscente (St. Tomás, 1 sent. $38,1.2 c$ ).

$Y$ aunque se diga que el universal en cuanto razón de universal está: sólo en el entendimiento, pertenece sin embargo por necesidad a las cosas que predica: manifestum est quod universalia praedicate... ex necessitate. insunt rebus, de quibus praedicantur (St. Tomás, In post. analy. 93). El realismo de los universales es aquí patente. Contribuye a esta actitud quizá la idea que se tiene de la ciencia. Si la ciencia ha de ser un conocimiento universal, necesario y de lo real, entendemos entonces la preocupación del pensador medieval (y también del antiguo) por concebir el conocimiento científico como expresión de la realidad misma.

En el Medioevo, en los siglos xI y xI, y quizá desde antes, se han dado también - como lo dijimos ya y como es harto sabido por lo demás- posiciones nominalistas. Pero no se registran brotes de esta tendencia después de la muerte de Abelardo hasta llegar al s. xIv. Lo que se debe quizá al fracasode los intentos de una explicación nominalista del lenguaje. ${ }^{7}$ La dificultad de

7 En el Medioevo se considera que la estructura del lenguaje (las categorias gramaticales) corresponden a la estructura de la realidad (a las categorias ontológicas). Las formas diferentes en las lenguas particulares son sólo variaciones accidentales de una común estructura. Los modistas - como se designa a los autores de gramáticas especulativas a partir del s. XII (de Pedro Helie en adelante) piensan que la "grammatica una et eadem est secundum substantiam in omnibus linguis, licet accidentaliter variatur" (R. Bacon, Gramm. Griega, cit. por Küng 1963 p. 14). Los modi significandi corresponden a los modi intelli- 
fundar una nominalismo en la Temprana Escolástica obliga a buscar la salida por el conceptualismo: este fue el intento de Pedro Abelardo. Pero el conceptualismo requería de una teoría de la realidad diferente de la metafísica neoplatónica, entonces vigente, $o$, en todo caso, de una actitud indiferente a esa metafísica. Abelardo optó por la segunda alternativa, pero al precio de un rotundo fracaso, como lo veremos. Asf́ pues, no sólo el nominalismo encontró dificultades insalvables para mantenerse, sino que tampoco el conceptualismo fue posible durante el lapso que corre desde la segunda mitad del s. XIr hasta los comienzos del s. xrv.

El nominalismo de la Temprana Escolástica tiene un origen dialéctico, vale decir lógico. Es el resultado del cultivo autónomo de ese arte liberal. Ahora bien, la lógica que se cultiva entonces es nominalista y no de conceptos. En esto se sigue a Boecio, quien por lo demás en este punto es tributario de la lógica estoica. Otro factor determinante de la actitud verbalista de los dialécticos es el cultivo que ellos hacen de la gramática. De manera que la Lógica de los medievales es una Sprachlogik, como la han denominado los alemanes. Si se repara por otra parte que en el famoso planteamiento del problema de los universales por Porfirio los géneros y las especies no tienen el carácter ontológico que en Aristóteles, sino que son términos relativos, esto es categorias puramente lógicas, se ve entonces que un cultivo de la dialéctica en forma predominante - como se hacia en algunos centros medievales de entonces - no podía menos que llevar a posiciones nominalistas. Esbozos de posiciones nominalistas advertimos ya en Eric d'Auxerre en el s. Ix y en el Comentario a Porfirio del Pseudo Rábano (s. x). Pero es Roscelino (1050-1120) el primero que habría afirmado que los géneros y las especies no son más que meras voces, más precisameńte flatus vocis (soplos de la voz). De Roscelino nada se sabe por su propia mano, con excepción de una carta agresiva pero sin importancia a Abelardo. Sabemos de su doctrina por Anselmo de Aosta, Abelardo, Juan de Salisbury y los epigramistas de la época. Por el primero sabemos que Roscelino habría afirmado que en la realidad sólo hay individuos y que las cualidades son sólo accidentes que acaecen en los individuos. Sería caricaturizar el nominalismo de Roscelino si se pretendiera que éste afirmó que las voces universales no significan nada. Todas las voces: predicados, sujetos, universales, singulares, géneros y especies están en el mismo plano. ${ }^{8}$ Ahora bien, es ridículo decir que el nombre singular no

gendi y a los modi essendi. Dentro de esta concepción es difícil fundamentar una tesis de significación que repose sólo en estructuras lingüísticas como productos de una institución cultural (humana). Tal fue sin embargo el intento de Abelardo, como se verá más adelante. (Cf. Küng, Ontologie u. logistische Analyse d. Sprache, Wien 1963, p. 14. Roos, Die modi significandi des Martinus de Dacia, Münster, 1952, pp. 151-2.)

8 Una exposición serena de esta doctrina se halla en el texto anónimo De generibus et speciebus, escrito probablemente a mediados del s. xII. Reiners: Der Nominalismus in der Frühscholastik, Münster, 1910, ha llamado la atención sobre este texto para informarse mejor 
designa nada: los singulares denotan individuos: de pareja manera las especies y los géneros señalan conjuntos, colecciones de individuos. Con ello no se quiere decir que los conjuntos, las colecciones, sean géneros y especies. Esta última es una posición realista bien conocida en el s. $\mathrm{xu}$ y que Juan de Salisbury la atribuye a Joscelino, obispo de Soissons. Esta es la famosa tesis de la Collectio que en los últimos tiempos ha sido actualizada y modernizada por Lešniewski.

Pará Roscelino en cambio los géneros y las especies, vale decir los universales no son ni están en los individuos ni en las colecciones o conjuntos de individuos, sino que son voces que señalan conjuntos de individuos. A la doctrina de Roscelino más conviene pues el distintivo vocismo que nominalismo.

El nominalismo o vocismo de Roscelino establece una relación directa entre las palabras y las cosas. Las palabras no significan contenidos mentales, como en la tradición aristotélico-escolástica, sino las cosas mismas. De manera que esta doctrina no sólo ignora lo que es el concepto, como el contenido mental al que significa la palabra universal, sino que hasta pasa por alto toda significación que tenga su base en el entendimiento (intellectus). De suerte que la base significativa de las palabras son las cosas, sean individualmente consideradas o en conjuntos (collectionis). ${ }^{9}$

Abelardo fue discípulo de Roscelino y de él recibió sin duda las ideas básicas de su doctrina nominalista, concretamente estas dos: $I$ ) el universal es una vox, 2) el género, la especie, el universal y el singular son categorfas lógicas, en consecuencia no podrían ser cosas ni estar en las cosas. El punto de partida del análisis abelardiano es pues lógico. Pero la critica mordaz al maestro Roscelino no se deja esperar. La vox es un producto tan natural como las cosas y es en cierta manera una cosa. Si se acepta para empezar la definición tradicional de universal: lo que por naturaleza se predica singularmente de muchos (natum praedicari sigillatim de pluribus), es obvio que la vox a secas no conviene a la definición de universal. Primero porque una cosa no se predica de otra. Y segundo porque la vox como sonus no se pronuncia idénticamente en cada caso en que se predica. De esta suerte el universal no es la simple voz sino la significación que está en la voz como en su vehículo. A esta vox significativa es a lo que Abelardo llama sermo. La diferencia que

de la tesis de Roscelino. El siguiente pasaje de De generibus... es ilustrativo: "De generibus et speciebus diversi diversa sentiunt. Alii namque voces solas genera et species, universales et singulares esse affirmant, in rebus vero nihil horum assignant." (V. Cousin, Ouvr. inéd. d'Abélard, p. 513. Cf. también Reiners, op. cit., pp. 24-26.)

9 Desde Aristóteles se hace la distinción entre nombrar (designar o connotar) y significar. Se nombran, designan o connotan las cosas concretas, pero se significan los contenidos mentales que están por las cosas concretas. Esta distinción se ve con más claridad en Boecio, In librum Aristotelis de interpretatione Commentaria minora. El vocismo no se detiene en la significación. 
hay entre vox y sermo es la que hay entre un producto natural y una institución humana: quid anim aliud est nativitas sermonum sive nominum, quam hominum institutio? (Nostr. pet $5^{22}{ }^{17}$, ed. Geyer.) Lo que se hace ad placitum, esto es por el hombre, es la unión de una vox a una significatio. En este punto no parece haber empero novedad con respecto a la tradición aristotélico-boeciana. Pero en punto a lo que es la significación la hay sin duda.

La significación para Aristóteles como para Boecio y los lógicos del s. xIII, William de Shyreswood por ejemplo, es el contenido intelectual que en este caso es la passio animae. Para Abelardo, en un primer momento, la significación es indesligable de la voz y no parece fundarse en un contenido intelectual sino en la función predicativa del sermo. El universal es un sermo praedicabilis. De esa manera el sermo es en cierto modo un syncategorema cuya significación estaría dada por su predicabilidad de muchos individuos pero singularmente cada vez. $Y$ aqui se descubre una nota nominalista de la doctrina de Abelardo. Dicho sea de paso, la expresión nominalista fue acuñada para tipificar la doctrina de los universales de Abelardo. (Cf. Juan de Salisbury, Metal: II, 17, y Polycraticus VII, 12). A principios del presente siglo Reiners (op. cit.) demostró brillantemente el carácter nominalista de la doctrina de Abelardo y negó que éste hubiese tenido una idea del concepto y, en consecuencia, que hubiera podido ser conceptualista. Pero Reiners no tuvo conocimiento sino fragmentario de los comentarios o glossae de Abelardo que editó B. Geyer recién entre 1917 y 1938. La obra de Reiners es de 1910. Por las glossae sabemos que el universal no se agota en su función predicativa, esto es como sermo praedicabilis, sino que por su significación se refiere a algo abstracto y unitario al que conviene un nombre propio significante de ese ente abstracto: sunt igitur genera et species in sensibilibus posita per appellationem, extra vero per significationem. (Nostr. Pet. $5{ }^{27} 7^{27}$ ). Esto que por su significación unitaria no está en las cosas sensibles es en realidad el concepto. Analicemos el siguiente pasaje sacado de la Lógica Ingredientibus para asegurarnos de ello.

La razón parece convenir con la autoridad en que los nombres universales designan formas comunes concebidas por el entendimiento cpues. qué otra cosa es concebirlas por los nombres que significarlas por ellos? Pero desde que nosotros sostenemos que estas formas concebidas no son lo mismo que los actos de conocerlas, ${ }^{10}$ hay en consecuencia además de la

10 "cum eas ab intellectibus diversas facimus" ha sido traducido por Gandillac, Oeuvres choisies d'Abélard, París 1945, p. 115, por “"puisque nous considerons que ces forms sont distinctes du contenu méme de nos intellections..." Esto es, ha traducido ab intellectibus por "contenido de nuestras intelecciones" con lo que además de tornar ininteligible el texto (¿cómo podría distinguirse entre contenido de conocimiento y objeto de conocimiento?) lo ha desvirtuado profundamente; ad intellectibus hay que traducirlo -como lo indican el sentido del contexto y las argumentaciones que preceden al pasaje citado- por 
cosa real y del acto de entender un tercer factor: la significación de los nombres. Que aunque la autoridad no lo sostenga, no es contrario a la razón (Ed. Geyer, p. $24^{23}$ ).

Este pasaje lo hemos reproducido in extenso porque es revelador en muchos aspectos del pensamiento abelardiano y porque con frecuencia ha sido mal interpretado y peor traducido. Está claro aquí que el nombre universal significa la forma común concebida por el entendimiento (conceptas communes formae). Pero esta forma no es ni la cosa real -a la que sin embargo de alguna manera refiere - ni es el acto de entender (intellectus actio). En efecto, así como el sentido no es la cosa sentida, el entendimiento no es la forma de la cosa que concibe (sicut autem sensus non est res sentita, in quam dirigitur, sic nec intellectus forma est rei quam concipit. Ing. $20^{28}$ ).

Ahora bien, el entendimiento, que en el proceso del conocimiento es el acto de entender, se diferencia de la forma que concibe, porque ésta es una cosa imaginaria y ficticia (res imaginaria quaedam est et ficta, loc. cit.) y el entendimiento es una cosa real, que no podría ser actualizado por una cualidad o forma ficticia. Abelardo entonces reclama que si la cualidad o forma conocida es ficticia, la sustancia de esta cualidad tiene que ser también ficticia (restat igitur, ut sicut ficta est qualitas, ficta substantia sit ei subjeta, Ing. $21^{13}$ ). De esa manera pues entre la cosa real y el acto de entender hay un tercer factor que es la forma o el concepto. Esta tesis de Abelardo es insólita en su época y, como él mismo nos lo dice, no es sostenida por ninguna autoridad. (Quod etsi auctoritas non habet, rationi tamen non est adversum), lo que en el siglo xIr y en el siguiente equivale a estar en la zona peligrosa de la oposición y la herejía. En el s. xirl en que la filosofía escolástica sigue, en punto al problema del conocimiento, a Aristóteles, la tesis de Abelardo fue ignorada. Para el Estagirita - y en consecuencia para la tradición aristotélico-escolástica_ el contenido del conocimiento intelectual se confunde hasta la identidad con el acto de conocer.

El concepto o forma es para Abelardo un objeto intencional que orienta el acto intelectual [forma vero in quam (intellectus actio) dirigitur: Ingr: $\left.20^{30}\right]$. Los escolásticos del s. xiv llamaron al concepto esse objectioum, pero por ser el objeto del acto intencional del entendimiento le llamaron también esse intentionale. De manera que Abelardo más se emparenta con los pensadores del s. xIv, del siglo de la decadencia de la Escolástica, que con los de su

"actos de conocer". Así lo ha entendido por ejemplo, Walter, Medieval philosophy, from St. Augustin to Nicholas of Cusa, N. Y., 1969, P. 198, quien ha traducido el pasaje en cuestion de la siguiente manera:

"But since we hold that these forms conceived are not simply the same as the acts of knowing them." Angel Cappelleti, Abelardo, Buenos Aires, 1968 p. 73, quien ha seguido puntualmente a Gandillac en su versión al castellano de un fragmento de Ingredientibus, según propia confesión, ha cometido los mismos errores de traducción que el guía. 
época y los del s. xir. Abelardo es el primer pensador del mundo antigtuo y medieval que distingue claramente entre el acto psicológico del conocer y el objeto ideal al que apunta. Al identificar la significación del universal con el concepto (fictum) Abelardo es manifiestamente un conceptualista.

El conceptualismo de Abelardo se halla sin embargo viciado por una metafísica que le es incompatible. No obstante que por un lado sostiene que los géneros y las especies son categorías lógicas, asume por otro, siguiendo a la tradición neoplatónica-cristiana, que Dios ha creado el mundo en géneros especies e individuos y que el concepto o forma es sólo un sustituto, un conocimiento provisional o hipotético de una realidad que no podemos conocer directamente. Pero no sólo esto. En la explicación del proceso del conocimiento normal introduce una teoría de la abstracción que en nada o muy poco difiere de la de Sto. Tomás de Aquino. "Si considero este hombre como substancia o como cuerpo - dice Abelardo- dejando de lado el hecho de que es animal, hombre o gramático no inteligo una propiedad que no tiene, pero no todas las que tiene... Y cuando digo que mi atención se dirige solamente a este ser en tanto que posee tal cualidad, la restricción concierne sólo a mi atención, no al modo de existir del ser... uno es el modo de entender y otro el modo de existir (quod alius modus est intelligendi quam subsistendi)" (Ingr. $25^{24}$ ). Es obvio que según esto el concepto no es una ficción sino una realidad abstraída, una forma que conviene a muchos individuos pero que es al fin de cuentas una forma de lo real. De esta manera, el Peripatético palatino no parece conceptualista sino más bien realista, al modo de Sto. Tomás de Aquino.

De Abelardo no se podría decir pues sin más que es nominalista, conceptualista o realista, porque, como hemos visto, su pensamiento se puede interpretar de diferentes maneras según los pasajes que se escojan. Estamos frente a un pensador no sólo complejo sino contradictorio. Rérmusat, conocedor profundo de Abelardo, ha escrito de él: "Faut-il admettre, en effet, ce vaste et incohérent ensemble de doctrines dans la tête d'un seul homme, et la philosophie d'Abélard est-elle le chaos?" 11

Creemos nosotros que las contradicciones patentes en el sistema abelardiano se deben antes que a las limitaciones personales de Abelardo, a su conflicto con la estructura cultural de la época. Abelardo no quiso o no pudo armar una metafísica diferente a la neoplatónico-cristiana que respaldara su posición nominalista o conceptualista. Lo que quizá hay que atribuirlo menos a incapacidad filosófica cuanto a la dificultad de ser cristiano en otra forma que como lo exigía entonces la autoridad y el dogma. Abelardo fue en primer lugar un lógico y el método empleado para inquirir sobre la naturaleza de las estructuras lógicas fue el lógico-lingüístico. $\mathrm{Y}$ con este método se llegaba inevitablemente a conflictos con la metafísica tradicional: los géne-

11 Abélard, París, 1845 , t. I, p. 273. 
ros y las especies no podía considerárseles sino como son: términos lógicos, pero de ningún modo elementos constitutivos de la realidad. ${ }^{12}$

Hay dos méritos de Abelardo entre otros que quisiéramos resaltar: r) Formula una teoría de la abstracción tan coherente y precisa como no volvemos a encontrarla hasta Sto. Tomás de Aquino. 2) Es indudablemente el primer pensador que tuvo un saber cabal acerca del concepto.

En los albores del s. xIv se dan los primeros planteos conceptualistas después de Abelardo. El franciscano Ricardo de Mediavilla distingue entre un universale in repraesentando o species y un universale in praedicando. En tanto que el primero es el acto real de entender (esse reale) el otro es un objeto de la mente (esse objectivum). Aquí vemos una separación clara de lo que estaba confundido en la teoría aristotélico-escolástica de la abstracción. El acto de entender tiene su objeto no en las cosas exteriores sino en un ser mental que deviene ser objetivo y cuya unidad lógica está determinada por su función predicativa.

Se han clasificado los conceptualismos que van a florecer a partir de este momento en lógicos y psicológicos. Aunque la denominación de conceptualismo psicológico es ambigua —en verdad el conceptualismo psicológico es el nominalismo de Ockham y los ockhamistas ${ }^{13}$ _ usamos sin embargo la clasificación por razones metodológicas. Hay pues el conceptualismo que afirma que el universal es un esse objectivum o fictum. Y hay el que sostiene que es el acto mismo de entender, la passio animae. Ockham se inclina, aunque

12 De Rijk sostiene en forma convincente que la "concepción de Abelardo sobre la naturaleza de los universales es sólo una particularización de su visión general" de las estructuras lógico-lingüisticas. La Lógica para Abelardo es ars sermonicalis. (Abelardo, Dialectica, Assen, 1970: Int. by L. M. de Rijk, pp. Xcv-xcvir). Cf. también Jolivet, Arts du Langage et theologie chez Abelard, París, 1969.

23 Los lógicos modernos llaman al conceptualismo escolástico, conceptualismo psicológico, por oposición al conceptualismo constructivista de data reciente. Cf. Stegmüller, Das Universalienproblem einst und jetzt, Darmstadt, 1965, pp. 48-118. Pero con esto se simplifica el problema al punto de oscurecerse líneas diferenciadoras que son empero muy perceptibles al conocedor de la Escolástica. No se ve bien cómo a los conceptualistas escolásticos, que reconocen un ente objetivo e ideal, opuesto y en cierto modo trascendente al acto de entender, pueda convenirles el adjetivo "psicológicos". Por otra parte, el ockhamismo no es conceptualista -y menos conceptualista psicológico, como lo quiere Stegmüller, aunque en esto coincidiendo con conocedores de la Escolástica, como Michalski, por ejemplo. El ockhamismo llega a posiciones nominalistas netas al rechazar el ser o la existencia de entes abstractos de cualquier indole. Küng (Ontologie und logische Analyse der Sprache, Wien, 1969, p. 11), él mismo un lógico notable, ha llamado la atención sobre el prejuicio de los lógicos modernos de concebir "las descripciones fenomenológicas de los conceptos objetivos como una aberración psicológica que debe ser superada. Estos prejuicios son sin embargo inconsecuentes, si se tiene en cuenta que son de quienes aceptan o adoptan clases intensionales, lógicas modales y relaciones de contenido. Trascribo a continuación el pasaje de la obra de Küng porque es sumamente ilustrativo: "Obwohl es logistische Kalküle nicht nur der extensionalen Umgen und der Modallogik gibt, so wollen sie meist nichts von der traditionellen Analysen der Wesenszusammenhänge wissen und halten die phänomenologischen Beschreibungen von objektiven Begriffen für eine pshychologische Verirrung, wie sie heutzutage überwunden sein sollte". 
titubeante, por esta segunda alternativa. Es sin embargo el conceptualismo lógico el que goza de más adherentes, aunque las críticas de los ockhamistas (de Jean de Mirecourt por ejemplo) manifestarán pronto las debilidades de ese conceptualismo.

Los dominicos Herveo Natal y Durando de Saint Pourcain ( $\left.\dagger 133^{2}\right)$ conciben un esse objectivum como opuesto funcionalmente a un esse subjectivum. Aunque ambos son esse in intellectus el segundo es múltiple según los diferentes entendimientos y los diferentes tiempos, pues se trata del acto psicológico de entender. EI esse objectivum en cambio no se "plurifica" en la variedad de las mentes y actos de entender: permanece uno y el mismo no obstante ser dependiente de la mente. Aunque no tiene un ser real en otro ser real, y aunque no es accidente ni substancia, esto es, no es reductible a ninguna de las categorías aristotélicas, tampoco podría decirse de él que es nada. El esse objectivum tiene la naturaleza de un esse ideale. Y en la medida en que corresponde a la intencionalidad del acto de entender es también un esse intentionale.

La línea franciscana del conceptualismo tiene otra evolución, en la que se percibe la influencia del augustinismo. Jacques d'Esculo y Pedro de Aureoli $(\dagger 1322)$ son figuras relevantes de este movimiento. La distinción entre esse subjectivum y esse objectivum es aquí más amplia. Para Pedro de Aureoli, el Doctor Facundus, el esse objectivum es el esse apparens, al paso que el esse subjectivum es la species. El esse subjectivum o species está causado por la cosa mediante la participación del entendimiento agente. El esse apparens no es efecto de la species pero sigue a ésta en una secuencia no temporal aunque necesaria. Ahora mismo, el Doctor Facundus distingue formalmente entre species y acto de entender, pues el esse objectivum no podría ser posterior al acto de entenderlo o comprenderlo: realmente empero la species y el acto de entender son lo mismo. Pedro de Aureoli mantiene la tesis aristotélico-tomista de la función activa de las formas de las cosas en el entendimiento, y hasta el recurso del entendimiento agente como elemento precipitador de la actividad intelectual; pero rechaza la pretensión de concebir la especie como medio de conocimiento. La especie es realmente-como dijimos- el acto de conocer pero no se conoce la cosa misma sino el esse apparens o fenómeno. El divorcio entre la causa efectiva del conocimiento y lo que realmente se conoce, entre la cosa o esse reale causante del conocimiento, y el esse apparens o fenómeno que es el objeto que se conoce, llevó inevitablemente al escepticismo. El Doctor Facundus examina hasta cuatro tipos de apariencias o fenómenos sin causa real. Algo más, considera la posibilidad de que Dios, como causa primera, cause representaciones de cosas realmente inexistentes no obstante las características de evidencia de ellas. Esta idea, que recuerda al "genie malin" de Descartes, la encontramos otra vez, pero ya dentro de una metafísica totalmente renovada, en Guillermo de Ockham. 
El conceptualismo psicológico está formulado por Ockham como una entre tres posibilidades de solución al problema del universal: el universal como fictum o esse objectivum, el universal como cualidad del alma, y el universal como passio animae. Aunque sostiene en algún momento el conceptualismo lógico, esto es el universal como fictum, Ockham parece inclinarse al final por el conceptualismo psicológico: el universal como passio animae. Quizás influyeron en esto dos razones:

r) El conceptualismo lógico podría devenir fácilmente -como en efecto sucedió en la filosofía racionalista de la época moderna- en un realismo de corte platónico. Si se opone el esse objectivum, como lo permanente e invariable, al esse subjectivum o acto de entender que se inscribe en el tiempo y es de naturaleza efímera, no se ve cómo podría explicarse esta relación si es que no se postula la real trascendencia del esse objectivum. 2) Se advierte de otro lado que la distinción entre fictum y passio animae es reductible a cero. $\mathrm{Si}$ al universal se le concibe como la intentio secunda animae, que por naturaleza signa muchas cosas de manera unívoca, como una predicado universal, no se ve la exigencia de recurrir todavía a un ente abstracto para que explique esta función de predicación universal. Para esta reducción vale la aplicación de la regla aristotélica-ockhamista: entia non multiplicanda sine necessitate. Jean de Mirecourt, cisterciense francés y ockhamista por añadidura, dijo que las distinciones entre esse subjectivum y esse objectivum son puramente metafóricas: omnia ista verba sunt metaphora. ${ }^{14}$

Pero el universal con ser para Ockham una passio animae no es, ni con mucho, species. Es sólo un signo que tiene con la cosa que señala o connota la misma relación que hay entre el humo y el fuego, entre el gemido y el dolor. La semejanza que pudiera haber entre las cosas y el universal que les conviene es la de causa y efecto, pero de ningún modo la semejanza por imago.

No obstante las variantes del conceptualismo escolástico podemos registrar los siguientes puntos concordantes: r) El universal está en la mente, es un esse in intellectus. 2) Su función es propiamente lógica y su unidad está determinada básicamente por su función predicativa: esse praedicando. 3) No es species intelligibilis ni derivado de ella: es más bien un signo o un ente ideal.

Aunque tener una idea del concepto no implica necesariamente ser conceptualista (Ockham stricto sensu no es conceptualista), no se es conceptualista si no se tiene idea del concepto. Ahora bien, es incuestionable que los conceptualistas escolásticos del s. XIv fueron los primeros -después de Abelardo- que supieron acerca del concepto.

Nuestra segunda pregunta era ¿̇en qué condiciones es aceptable el concep-

14 Cit. por Michalski, Les sources du criticisme et du scepticisme dans la philosophie du xiv siècle, Cracovie, 1924, p. 24. 
tualismo en la Edad Media y el saber del concepto? Vimos que el intento de Abelardo para justificar su conceptualismo fue históricamente frustráneo: no tuvo eco en las generaciones siguientes y su misma posición teórica acusa ambigüedades y contradicciones. Habíamos anotado que ello se explica porque el planteamiento del problema se hizo sólo desde el punto de vista lógico y gramatical y no se concordó las soluciones con una posición filosóficamente individualista. Abelardo aceptó la metafísica tradicional y se basó en la igualdad de las cualidades de las cosas para explicar el concepto, con lo que llegó anticipadamente a las soluciones realistas del tomismo.

Ahora bien, todo esto no es casual. Hay un trasfondo metafísico realista de origen neoplatónico en el pensamiento escolástico, que actúa a modo de presupuesto en toda posición teórica: el universo es un conjunto ordenado de cosas que se relacionan esencialmente entre sí, como que es el producto de una inteligencia divina. La categoría de "ordo" no se explica sino dentro de la perspectiva del Intellectus divinus. Aunque se afirma la existencia individual, incluso por exigencias del credo, el individuo sin embargo expresa una forma o esencia, cuya plenitud y desarrollo se alcanza en el entendimiento divino como idea ejemplar y en el entendimiento humano como abstracción. Pero esto supone también que la estructura de la realidad es plenamente inteligible no quoad nos, sino per se. No hay entonces oposición entre la fe y la razón sino que más bien la una se complementa en la otra. Por eso la directiva del pensamiento escolástico es: credo ut intelligam o fides quaerens intellectum. La fe no es una adhesión pasiva sino un movimiento de la inteligencia por penetrar en lo que se ha asumido oscuramente por provenir de la revelación y del testimonio de la palabra de Dios.

Dentro de este contexto no es posible o aceptable el conceptualismo. Como decíamos al comienzo, inquirir por las condiciones en que es posible el conceptualismo es también averiguar por las razones que explican la disolución del realismo medieval, del realismo de los universales.

Steinbüchel ${ }^{15}$ ha señalado dos movimientos iniciados ya en el s. XII, cuyo desarrollo minará la unidad de la cultura medieval: I) Los pueblos instruidos por la Iglesia y sujetos a su tutela cobran poco a poco independencia y conciencia de sf. 2) La cultura burguesa, que comienza a florecer en las ciudades, reemplaza a la cultura clerical. Con el comercio en las ciudades y el paulatino afianzamiento de los Estados nacionales se va generando una fragmentación del poder, a la par que se va quebrantando la unidad del poder de la Iglesia, símbolo de la idea de ordo. En el plano teórico se aprecian movimientos intelectuales conflictivos, sobre todo en las universidades. En estos centros de enseñanza se afirma - sobre todo en la segunda mitad del s. xIII - un individualismo cada vez más radicál y la autonomia del saber filosófico. Roger Bacon (1214-1292) es el primero que 
sostiene la validez ontológica del individuo y su singularidad esencial. No concibe cómo el individuo ha de explicarse a partir de la forma por la participación accidental de la materia y no más bien al revés. Como se sabe, el aristotelismo tomista afirmaba que la materia est principium individuationis. En todo caso, sostiene Roger, el individuo no es un accidente de la forma ni se origina a partir de ella por la materia. Para el Doctor Mirabilis, si bien el individuo es un compuesto de materia y forma, lo es de una materia individual y de una forma también individual. Dentro de la esfera del conocimiento no se ve por qué el entendimiento no pueda conocer directamente y sin mediación de abstracción, al individuo mismo. La species individualis está en el entendimiento con igual rango que la species universalis. Aunque la posición de Roger con respecto de los universales es realista, su realismo se incribe en un contexto teórico enteramente diferente al tradicional y en el que se nota un acento individualista innegable.

Juan Duns Scoto (1270-1308) avanza un poco más por la ruta del individualismo. El individuo tiene una forma esencial propia y se diferencia por su esencia radicalmente de otro individuo. La forma individual que constituye su esencia (haecceitas) no es conocida in statu ipso, pero sí dentro del orden divino y beatífico. Esta concepción se funda evidentemente en un axioma teológico. En efecto, a nivel de la experiencia humana los individuos se distinguen sólo por sus accidentes sensibles. Se escinde así la unidad del saber. De un tajo queda separado el saber teológico del saber filosófico.

Esta concepción dual de la realidad tiene expresiones paradojales en la Facultad de Artes de París alrededor de 1270. En el Estatuto de la Universidad de París de 1272 se leen prohibiciones expresas de tratar en la Facultad de Artes asuntos concernientes a la fe. Pero cuando sean temas cuyo tratamiento fuese común a la filosofía y a la teología, "se privará perpetuamente de los derechos de pertenecer a nuestra sociedad a quien resolviere las cuestiones en contra de la fe". ${ }^{16}$

Esta restricción que ya entonces era asfixiante en la Facultad de Artes obliga a algunos filósofos a formular la teoría de la doble verdad. "La eternidad del mundo" y "la unidad del alma intelectiva" —decían los averroistas latinos, Sigerio de Brabante y Boecio de Dacia - son proposiciones filosóficas que sólo en apariencia contradicen artículos de la fe. La fe tiene su esfera propia que es la revelación. La filosofía se mueve empero dentro de la esfera de la razón. La teoría de la doble verdad fue condenada expresamente por la Iglesia. El obispo de París, Stephan Tempier emitió en 1277 un decreto condenatorio de 219 tesis entre las que figuran naturalmente

16 Statuimus insuper et ordinamus quod si quaestionem, aliquam, que fidem videatur attingere simulque philosophiam, alicubi disputaverit Parisius, si illam contra fidem determinaverit, ex tunc $a b$ eadem nostra societate tamquam hereticus perpetuo sit privatus. (Chartularium Univers. Parisiensis, vol. I, p. 499, n. $44^{1}$. 
las pertenecientes al averroismo latino, pero de paso -o aprovechando la ocasión-algunas tesis tomistas.

Vemos cómo se va separando el mundo de la razón del mundo de la fe y cómo se va reduciendo la teología al contenido bíblico, esto es a la sola revelación. Guilermo de Ockham (129o/5-1349) afirma que muchos artículos de la fe no son principios demostrativos ni demostrables. Algo más, a muchos sabios y estudiosos de la naturaleza les parecen falsos. Y según Ockham, ni siquiera es demostrable la unidad de Dios y su infinitud. La existencia de Dios sólo admite una prueba probabilística.

Pero Ockham es sobre todo un teólogo y quizá está acertado Vignaux cuando afirma que el nominalismo de Ockham sólo se entenderá dentro de la perspectiva de su teología, o no se entenderá. ${ }^{17}$ Su teología se basa en la idea de una potentia Dei absoluta, cuya limitación no estaría más que en el principio de no contradicción. Ahora bien, Dios no puede. hacer que los individuos sean distintos y comunes a la vez. Toda diferencia de razón o formal es una diferencia real. De modo que los individuos ya en la creación y desde la creación expresan su heterogénea y real individualidad.

El cuadro medieval de un todo ordenado e inteligible, expresión consecuente de un intellectus divinus, se resquebraja y da paso a la concepción de una multiplicidad de individuos cuyos ordenamientos contingentes están determinados más por la voluntad y el poder que por la inteligencia. Los designios de Dios son impenetrables no por inaccesibles, sino porque dependen de la voluntad divina, de la "potentia divina", cuya impredictibilidad es absoluta.

Se ve ahora claro que el conceptualismo es una teoria coherente con esta nueva manera de sentir y concebir la realidad. El universal es un abstracto, en el sentido ockhameano, y no una realidad. La validez de los conceptos lógicos reposa en la posibilidad de su confrontación cọn la experiencia y en la verificación experimental. Es fácil ver cómo se hace posible desde esta perspectiva la ciencia moderna, vale decir, las ciencias empíricas de la naturaleza. Pero el nacimiento y desarrollo de las ciencias empíricas van a dar lugar a otras metáforas filosóficas. La ciencia y sus éxitos han inspirado metáforas que han consistido _-según decir de Husserl ${ }^{18}$ _ en la identificación del método científico con la estructura de la realidad. El racionalismo y el empirismo de la época moderna son hijos de esa alegoria.

\section{UNIVERSIDAd MAYOR de SAN MARCOS}

LJMA

Antonio Peña Cabrera

17 Vixnaug, Nominalisme au XIVe siècle, Montreal, 1948.

18 Husserl, Die Krisis der europaeischen Wissenschaften und die transzendentale Phäenomenologie, Haag, 1962, pp. 62-66. Cf. también Blumenberg, Paradigmen zu einer Metaphorologie. En Archiv für Begriffsgeschichte Bd. 6, Bonn, 1960, p. 55 . 